\title{
Un viaje por la poesía de José Antonio Mazzotti: a propósito de El Zorro y la Luna. Poemas reunidos 1981-2016
}

A journey through poetry of José Antonio Mazzotti: about El Zorro y la Luna. Poemas reunidos 1981-2016

[Crítica y testimonio, a partir del premio especial de poesía José Lezama Lima otorgado por Casa de las Américas al libro mencionado en enero del 2018]

\section{Roger Santiváñez \\ Temple University, Filadelfia, Estados Unidos \\ Contacto:royika@hotmail.com}

Esta historia comienza en Lima, 1978 cuando conocí al joven poeta José Antonio Mazzotti (Lima, 1961). Ambos estudiábamos literatura en la Universidad Nacional Mayor de San Marcos. Lo conocí - podría decirse- antes de conocerlo: nuestro profesor, el poeta y novelista Edgardo Rivera Martínez, me habló de un joven cachimbo (como se denomina en el Perú a los ingresantes) que le había llamado la atención por su talento. Fue así que poco después tomé contacto con él una buena mañana en el Patio de Letras. Empezamos a frecuentarnos todos los días en la Universidad. De manera que, en cierto modo, fui testigo de la composición de su primer volumen Poemas no recogidos en libro, el cual obtuvo el primer premio de poesía en los Juegos Florales Túpac Amaru convocados por la Federación Universitaria de San Marcos (FUSM) en 1980.

En efecto, recuerdo nuestros vagabundeos por el centro de la ciudad de Lima, en compañía de los jóvenes de nuestra generación que ya se perfilaba y que tomará cuerpo de ciudadanía a principios de la mencionada década de 1980. De aquellos días cito: "Sólo supiste atesorar esos desnudos en los almanaques, José Antonio, / y caminar por La Colmena puesto que el día y la noche apenas si te eran propicios / para buscar el amor" (23). Estos versos pertenecen al poema "Canto a mí mismo", que -con lejana resonancia withmaniana y autoirónica alusión a un conocido valse peruano de Chabuca Granda- se sitúa como un eje al centro 
del primer poemario de Mazzotti. El texto grafica claramente las preocupaciones de un adolescente de la clase media limeña, los prolegómenos de su aprendizaje sexual y, por supuesto, la ansiedad del amor en los momentos álgidos de aquella juventud -terrible y hermosa- que nos tocó vivir en el Perú en las primicias de la década que se iniciaba.

Esta dupla-la del amor y la ciudad-será el axis desde el cual se va a desarrollar Poemas no recogidos en libro (1981). Y en el meollo de dicha juntura, la escritura. Leamos del texto que abre el conjunto: "y descubres que un amor y un poema son lo mismo al fin y al cabo" (17) lo que llevará al sujeto poético a una suerte de encrucijada sin salida - proclive al suicidio- como sugiere el poema siguiente, "Contra el arte poética": "Apenas un juguete entre tus dedos (una Browning tan dulce como un lapicero)" (18). Es decir, el poeta se debate entre su intenso deseo de escribir, una cierta frustración en ello ("Ni siquiera has logrado conmover a un garabato" [18]) -lo cual entraña (desde Rimbaud) la famosa pregunta por el sentido o no de escribir poesía- y finalmente el reconocimiento general del absurdo de la existencia: "Has apelado a los dioses y no te han atendido" (18), aunque contradictoriamente se autoafirma el poeta en la persistencia y constancia del oficio: "y apenas si has logrado conmover a un garabato" (18). De todos modos algo se consiguió. La escritura misma. Y eso basta.

Así entendemos el poema "Oración”, que termina por negar la existencia de Dios o los dioses, pese a que está dirigido justamente a él. Con una dicción heredera del desenfado de la poesía conversacional -en pleno auge por los días de la composición de este poema- o más precisamente del Exteriorismo de Ernesto Cardenal, leemos: "Señor, / deja correr las aguas, / ciérrate la bragueta, vete a andar, y no hagas caso de lo que te digan, / tu pueblo apenas si conoce las carátulas mimetizadas" (19). Crítica a la alienación masiva de los kioskos periodísticos de la ciudad, cifrada en la irreverencia iconoclasta que, como veremos más adelante, será una de las características principales de la poesía de nuestro autor. Aquí Mazzotti paga tributo tanto al ateísmo materialista -de signo marxista- que 
alumbró los ideales de transformación revolucionaria de la generación a la que pertenecemos, como a la preeminencia del modo coloquial que presidía nuestra concepción poética en aquellos años de gran esperanza e inquietud.

Una atenta lectura de todo el libro arroja el resultado de una conseguida estructura organizada en torno a los tópicos ya mencionados: el amor y la ciudad, ambos entramados en el asunto de la poesia -la escritura- y una cierta encrucijada que se define ante la inutilidad del arte o negación del canto y simultáneamente del mundo y de la vida. Sin embargo, el poeta se encarga de dejarnos un resquicio de esperanza y reivindicación del oficio. Toda esta secuencia está sostenida en un fresco tono coloquial -resonancia de las lecturas de la época, principalmente de las generaciones del 60 y 70 peruanas-, asimilado con original cadencia personal: "Nosotros no inventamos el amor. Setiembre rojo. / No fuimos los primeros en remar bajo los puentes. / Ni siquiera en escribir sobre boletos de autobús" (27). Los parques de la ciudad serán el escenario recurrente, refugio de infinitas caminatas -obsesión del flâneur baudeleriano- y espacios privilegiados para parejas de amantes juveniles; pero también motivo proclive a una reflexión de tipo político: "y los parques enrejados donde se filtra el humo de las fábricas" (28). Este elemento político será -como veremos más adelante- otro tema recurrente en la poesía de Mazzotti. "métete la realidad en el poema" (21) es una clara recomendación que leemos en el poema "A un joven poeta activista".

Claro que este es uno de los postulados centrales del conversacionalismo latinoamericano en que nuestra generación nació a la poesía. Pero en el contexto peruano significaba un tema de discusión a la orden del día, liquidada la experiencia reformista realizada por la denominada que lideró el general Juan Velasco Alvarado entre 1968 y 1975. En efecto, el golpe fascistoide del general Francisco Morales-Bermúdez -el, como lo llamó el famoso historiador Jorge Basadre- procedió al desmontaje de las reformas, lo que desencadenó una crisis socio-económico-política cuyo punto culminante fue el Paro Nacional Unitario del 19 de julio de 1977. Esta situación provocó un álgido debate en torno a las delicadas relaciones entre marxismo y poesía; y -smultáneamente- atravesó la 
textualidad del poema, como podemos verlo en este verso: "y el lomo en que cabalgo rodeado de metrallas y sirenas anunciando un bombardeo" (25). Fiel retrato del ambiente militarizado y represivo de la dictadura gorila de MoralesBermúdez y también llamado de alerta a lo que pronto dominará la escena política peruana: la guerra desatada por el Partido Comunista del Perú en 1980.

Quizá esta atmósfera de violencia y muerte cercana es lo que alumbra los versos finales del "Canto a mi mismo" citado al principio de este artículo: "Sólo caminas como un árbol desteñido / y escuchas a lo lejos una radio con un disco repitiéndote / cosas que quieres olvidar, un vals llorando sobre tu cabeza / y unas palabras para arrepentirte de tu reciente defunción" (23). Y en el poema que sigue a este, encontramos estos acordes que, en cierto modo, resumen el planteamiento de todo el libro: "dime muchacha si no es cierto el tiempo / dime si no es mejor andar hacia la muerte distraído / y odiar la poesía como a una cucaracha / nocturna en cuyo lomo el pie no acierta" (24). El texto final del poemario -significativamente titulado "Marcha fúnebre"- cierra este cómputo con una orquestación (no olvidemos que el epígrafe inicial reza el” de Huidobro) un tanto extraña al lenguaje coloquial imperante: "Muertos. / Estamos muertos. / Repetición inalterable de las olas. / Piedra sobre la piedra, luego arena / y nuevamente piedra" (29), que tal vez alude a la alienación cotidina, pero también al último desenlace al que estamos destinados -lo que está declaradamente dicho en los dos primeros versos- como condición metafísica de la existencia del ser; aunque una luz de esperanza -amorosa y humana- acierta a surgir en dicha oscuridad: "La timidez vencida por las olas, por la noche" (29). Es posible la relación fructífera entre las personas entonces.

El tema metafísico abre el libro Fierro curvo (órbita poética) (1985) con el innominado poema que empieza "Estas palabras (así en negritas) que recrea -a la manera de "La pieza oscura" de Enrique Lihn- una escena de la infancia. Aquí el sujeto poético es poseído por la memoria de una angustia radical sentida en la etapa inicial de su experiencia vital: "estar marcado por ese maldito signo en mi ascendente, ese maldito signo que me impide desarraigarme de / esa imagen en 
el espejo a los seis años, mirándome jugando, completamente solo ¿dónde están? ¿dónde / están?, y dejé el trencito en el suelo, no escuché nada, y yo sabía / que todo estaría friamente marcado por el resto de mi vida" (32). Esta sensación de vacío y soledad va a primar en todo el poemario, igual que en el siguiente Castillo de popa (1988) ambos conjuntos escritos a lo largo de los años, hasta agosto de 1988, momento en que nuestro autor deja Lima para trasladarse a los Estados Unidos y seguir estudios de post-grado. Debido a esta circunstancia temporal los analizaremos juntos.

Recuerdo vívamente los veranos de 1983 y 1984 en que se estrechó mi amistad con José Antonio Mazzotti y traigo a colación esta memoria porque fui testigo de la composición de lo que entonces se llamaba Libro del estado natural -texto originalmente independiente que luego pasaría a convertirse en la primera sección de la edición príncipe de Fierro curvo-. Pues bien, aquí en esta recopilación que comentamos, se recogen cuatro textos de aquella sección. El concepto es -en la particular concepción mazzottiana- una contradictoria condición que oscila entre la llana y sencilla realidad humana y -por otro lado- la imposibilidad del amor en tanto "retraso del estado natural" (33). Es importante manejar dicho concepto porque en los dos libros que estamos estudiando -Fierro curvo y Castillo de popa- el tema del amor irrealizable circula entre sus páginas como una especie de maldición (que quizá proviene de aquella marca de angustia impronta desde la más remota infancia, como señalamos algunas líneas arriba), pero la pena de amor -en cuya entraña vibra siempre el auténtico deseo de realizar ese amor- deviene pronto no sólo en una lucha íntima y personal, sino en la sincera preocupación por los problemas sociales de la realidad peruana, principalmente el sufrimiento del pueblo explotado y marginado, así como la reivindicación de un cambio revolucionario hacia la liberación de la persona humana.

En "Apassionata" de Fierro curvo podemos leer: "y lo único que queda / es niebla invisible / y ramas secas / lo único que queda / humo en los ojos / tu ombligo / en el centro / del cielo / el grito de un pájaro manco / esperando que 
el mundo se inciende" (37). Como vemos, estamos ante una suerte de ecuación entre la desolación amorosa y el deseo de la Revolución. A propósito de los problemas en la relación de pareja y -siempre en Mazzotti- su vínculo con la poesía (la lengua) cito estos versos de "Canción a una limeña" por la lograda magia de su ritmo: “Así dirás que te he olvidado, y será cierto / porque más fuerte olvida el que recuerda y no ama / que el que no ama ni recuerda / las letras de un idioma verdadero" (38). Incluso en un poema como "Dante y Virgilio bajan por el infierno", con todo el desarrollo intertextual que conlleva, descubrimos que Beatriz (Beti en el desenfado coloquial de Mazzotti) viene a ser la transfiguración de una musa real y es clave también -para el Ars Poética general de nuestro autor- del abandono, aislamiento y sarcasmo que va a significar el desamor, la vida y la poesía -todo junto-, como queda ilustrado en estos versos, ante el reclamo de ayuda por parte del poeta, al que Virgilio responderá: “Estúpido. ¿A qué te metes en ridículo negocio?"” (44), para luego enterarnos de que Virgilio "frotó su saquito / y desapareció en la multitud" (44), abandonando al poeta en el infierno (del desamor) peruano.

Este poema y los dos siguientes que cierran Fierro curvo comparten una onda literaria y mitológica. El primero, "Noche serena / Versión libre de la Oda VIIIa del fraile agustino Luis de León / Salamanca, 1580", testimonia el surgimiento de la guerra de -ocurrido en el Perú desde 1980-, pero esta situación esta transrealizada vía el mito grecorromano: "y puedo entonces dibujar entre las sombras cómo otro camino / prosigue el sanguinoso Marte airado: / en el cerro de Amancaes / donde suelen las teas chorrear la cabellera" (45). Alusión directa a los candiles que con el diseño de la hoz y el martillo hacía brillar en las noches de apagón en los cerros que rodean Lima. Pero la opción del poeta es por el amor: “Así, amada, reza / la antigua receta de la diosa impúdica, para quien / la belleza / debe ser deleitada" (46). El otro poema torna su interés a la historia andina prehispánica, vinculada a la mitología quechua. En una propuesta de reivindicación étnica habla Cuismancu, cacique de la región Lima, en la época del Inca Pachakutik: "soy el rey, / el rey / ordenador, embajador del cielo, intérprete de Rimaq, / hijo de Pachakamaq, padre y finalmente 
sujeto / a una extraña certeza..." (48). La certeza es que Cuismancu -o mejor dicho la raza que él representa- está vivo en la actualidad de indios y cholos que pueblan mayoritariamente la ciudad de Lima: "Soy yo. Cuismancu regresado" (48). Y luego: "vivo en los cerros / mirando el exceso estadístico / de construcciones deformes que hablan / de un dios que no se parece / en nada a sus palabras" (49), para terminar con el anuncio definitivo de una transformación que vendría como un torrente desde los Andes y arrasaría el sistema inaugurando un nuevo orden con un signo étnico muy claro: "Esas piedras / caerán por su peso / y un huayco / fundará con sus venas / chorreando un cuadro del crepúsculo / tamaño natural, cactus y jora" (49).

Esta aproximación cultista está tambien en Castillo de popa -en los poemas "Fábula de P. y G." (Polifemo y Galatea) y "Francesca / Infierno, V"-, ambos girando en torno al tema del amor, el anuncio de un edad futura mejor y una hábil traslación al tiempo presente ["Pancha (para los amigos)" (63)]. Pero el libro se estructura sobre la base de un viaje y su naufragio correspondiente, el cual se nos presenta desde el "Introito". Tenemos la imagen de un galeón -símil de la travesía- donde viaja la poesía hacia ningún lugar. Lo interesante es que el poeta enfatiza la tensión -que como sabemos es uno de los ejes centrales de la poesía de Mazzotti- entre la interioridad sentimental y la preocupación por la exterior historia socio-política: "será tu país ese que cantes / pero no tu corazón" (53) aunque contradicción esencial en toda gran poesía- podemos leer: "(Canta, canta, pato feo, / que todo lo que cantes quedará grabado / como el sueño de un pobre insensato / al derrumbarse las columnas de la villa" (53). Defensa de la poesía en tanto única posibilidad de cierta permanencia sobreviviente en medio del caos y la destrucción final. Negatividad implícita pero música de todos modos cuando todo acabe en definitiva: "El canto solitario / apesta a peces muertos. / Lo demás será mañana / comida de los cormoranes" (53). Sea como sea: allí está el canto.

El viaje de este peregrino incluye el valle del río Santa en la sierra norte del Perú, las pampas de Nazca, referencias a la selva amazónica (vinculada intertextualmente a una Bucólica de Virgilio) y -por supuesto- la costa marina 
cercana a Lima: del poema dedicado a ella "Pelícanos" quisiera destacar la lograda prosodia de estos versos "Sólo tablas / y huesos de cangrejo estallando en la orilla, sólo tablas / chillando en el agua y el chasquido de las piedras" (64). Habilidad que Mazzotti desarrollará en el manejo del clásico endecasílabo como veremos en libros posteriores. Pero vayamos a "El estanque". A mi juicio este texto constituye el punto nodal de Castillo de popa. Se abre con una cita de Cernuda, según recuerdo una de nuestras lecturas preferidas en la Lima de los 1984-85. El poema es una especie de paneo -siempre el viaje- por la historia y la sociedad peruanas, pero lo intersante es que todo está planteado desde una perspectiva que podríamos calificar de conceptual o - por lo menos- trabajada en sesgo. Es decir, no hay referencias directas al tema, sino imágenes de diversa índole que van dando cuenta -con una óptica extremadamente crítica- de la experiencia nacional en el país. Por ejemplo: “(Sopla un viento furioso en una parte del tiempo. / Hay indicios; queda todavía / una intuición" (66). O "(Me da miedo seguir: el manso pelaje del estanque dibuja un remolino / que es sólo su propio pasado: no llegar a ningún sitio / saltando al autobús en la mañana)" (67). Se constata una frustración nacional profunda: "Ser de aquí es ser de ningún sitio" (67) y el tono final de amarga melancolía despunta de todos modos la opción por la poesía: “(Quién sabe todo esto sea parte del paisaje. / Pero es bello dibujar / el reflejo de la luna sobre el agua)" en ese estanque -entendemos-que sería el Perú.

Cierro mi incursión en Castillo de popa con un poema que me parece de singular trascendencia. Está relacionado a la guerra civil habida en nuestro país en la década de los 1980s y significa un escenario más de la preocupación poética de nuestros autor por los destinos patrios. "Diuturnum illud / Sueño profético de Wanka Willka". Esta última es una voz quechua que sería el más remoto orígen de la palabra Huancavelica, nombre que designa uno de los departamentos más pobres de la sierra sureña andina y cuyo significado, según una antigua versión, es Piedra sagrada. Pues bien, me es grato recordar que mi amigo José Antonio Mazzotti pasó una breve temporada -en el verano de 1980- en estas cordilleras. De esa experiencia data el poema que aqui comentamos. Es un retrato fidedigno 
de una comunidad campesina de las alturas y de su preparación para la insurgencia armada bajo la dirección de, ante la atenta mirada de los comuneros: "Tú sigue craneando tu informe, gringuito..." (72). Y más allá de la comprobación de la extrema miseria de la zona -que indigna y rebela al poeta- está el sentimiento de aislamiento que prima: "Y me sentí más solo / que un pobre riachuelo de la puna" (72) aun cuando hay una solidaridad explícita por el resultado de la posterior violencia: "Los últimos restos del poblado / como un cuerpo anestesiado quejumbraban / la muerte de Felícita y los hijos, el exilio / a las pampas amarillas de la costa" (72) también se nos ofrece la amarga evidencia del sinsentido de la guerra: "serían demasiados los cadaveres / y pocos los frutos inmediatos. En resumen: / una pésima inversión" (73) porque -al final- parece quedar sólo el signo de la destrucción y nada más: "Crepitan rescoldos y un gemido subterráneo. / Una inmensa pradera de cenizas se confunde con el mar" (73).

En agosto de 1988 el poeta viajó a los Estados Unidos para seguir estudios de postgrado, empezándolos en Pittsburgh para obtener su doctorado en Princeton University con una tesis sobre el gran cronista mestizo Inca Garcilaso de la Vega. No nos sorprende entonces que el gran tema de su primer conjunto -escrito en los Estados Unidos y titulado El libro de las Auroras Boreales (1994)- sea la nostalgia de la patria lejana. Pero - como como ya hemos ido viendo- el tema de la lírica en sí misma es el que abre esta breve colección. Y profundamente autocrítico entronca el poema liminar -denomimado "Invitación"- al concepto que viniendo desde Rimbaud y su famosa renuncia a la escritura se enlaza con el gran poeta chileno, estrella de la constelación conversacional latinoamericana de los 1960s: "lo que Lihn había dicho: / podemos defecar lujosamente / sobre la poesía" (80). Esta suerte de amor-odio prosigue con el texto "Harakiri con pluma" de clara connotación -diremos- suicida antipoética, pero con la salvedad, ya permanente en la obra de Mazzotti, de -aunque sea a regañadientes- mantenerse en la poesía. Dicha íntima vivencia y convencimiento parece cobrar sentido armas de la literatura- en la memoria de la infancia como el paraíso perdido, espacio de la felicidad desaparecida. Así tenemos el poema "Los padres" cuyo 
emotivo remate nos exime de mayor explicación sobre el punto. Recordando un día de playa nos dice: "el instante en que él me levantaba de una ola siniestra / y ella acudía sonriente / toalla en ristre / tumbando al atrevido infante / bajo el halo de sus plumas tibias" (82). Partiendo de esta imagen Mazzotti se conecta con el asunto nostálgico. Situado en los Estados Unidos -específicamente en Manhattan en un caso-el sujeto poético es poseído por lo que llama "las aldeas del recuerdo" (83), estado en el que diseña unos endecasílabos perfectos: "y la espina boreal de los collares / apenas si repinta un trazo fresco / a los rostros andinos que aparecen" (83). Como podemos observar se trata de una dicción -hasta cierto punto alejada del conversacionalismo estándar- que presenta una otra realidad puramente verbal para tocar la referencia directa. Creemos que este rasgo de la poesía mazzottiana se va perfilando cada vez más, lo que no le impide momentos de abierto coloquialismo: "El Perú me visita" (85), y más adelante, "Escribir a tus espaldas es lo que me queda. / Seguir tu rastro inerte como un ebrio / que chanca la noche y chanca" (85). A veces, nos trituran los recuerdos.

El siguiente libro escrito por José Antonio Mazzotti en los Estados Unidos se titula Señora de la Noche (1998). Esta compacta colección, originalmente editada en México bajo el sello El Tucán de Virginia, principia con una interesante sección, "Gimnasio de papel”. Los textos giran en torno al ejercicio físico, y allí se compara dicho entrenamiento con la propia poesía: ambos esfuerzos aparecen como inútiles y absurdos finalmente, salpicados de alguna pincelada erótica o de una cierta identidad andina reivindicada por el sujeto poético. Cabe destacar que en esta parte figura "Al fondo del jardín", uno de los más celebrados poemas del autor, así como -en la segunda parte- "Eclipse de noche", "Una inmensa flor de belladona" y "Como pétalos abriéndose en la noche", cuyo virtuosismo en el manejo del ritmo -algunos grupos de prosódicos endecasílabos- es notable; así como -en el tecero de los nombrados- en la moderna elaboración del clásico tópico del Carpe Diem. Esta aseveración nos lleva a señalar a Marte, Adonis, Venus "diosa de nácar" (112) y la Ninfa frente al navegante, que como tal -o pasajero, ya sabemos- se identifica el sujeto de estos versos. Pero del guiño con 
los clásicos Mazzotti salta a la vanguardia, si no, veamos esta re-creación ultraísta: "y el sol / chillando en todas las ventanas" (117). Hablando de lo moderno o de la modernidad, centrémonos en la composición final del conjunto "Enrique de Ofterdingen envía postal después de unos años" homenaje a Novalis y preclaro Arte Poética del autor. Es un reconocimiento -a través de la apropiación de la voz del gran romántico- a la condición del ser poeta. Desde la comprobación de la trágica finitud de nuestra existencia y el dulzor contemplativo de la misma, el anhelo por alcanzar la Flor Azul -símbolo de la utopia poética- el creador no cesa en su búsqueda incesante por ese "diamante negro" (118) que le fascina "esculpirlo con la lengua / arriesgar el futuro y la reputación / con paciencia de fabbro paduano" (118). Extrema defensa poundiana del verso cincelado en el lengauje que -como vemos a cada tramo de su obra- va siendo la última y primordial opción de nuestro poeta.

Declinaciones latinas es el libro que sigue en esta recopilación y está fechado entre 1995 y 1999. Podemos suponer entonces que fue escrito en estos años cuando periclitaba el siglo $\mathrm{XX}$, quizá por eso tiene un sentido de balance y liquidación de una etapa. La preocupación central del poeta es la reflexión sobre los destinos de la nación peruana -lo que se llamó en el Perú desde los días de José Carlos Mariátegui [1920s y 30s]- reflexión que ocupó lugar preeminente en el pensamiento peruano hasta bien entrada la década de los 1980 s, cuando al ritmo de la caída del muro de Berlín el mundo pasó a otra etapa histórica.

Este libro de Mazzotti parece querer incidir - poner su grano de arena desde la poesía- en aquella álgida discusión finisecular ad portas el siglo XXI. Partiendo de una gran incertidumbre con la que nos inicia el viajero poeta su aventura, nos asomamos a los críticos y dramáticos textos de dos conjuntos: "Himnos nacionales" y "Declinaciones latinas", sendos poemas constituidos cada uno por diez cantos. Los dos grupos se complementan. El primero de ellos se diseña como una suerte de canto generacional, en medio de una terrible confusión y desconocidos futuros anhelos. Los versos juegan con la letra del himno nacional 
del Perú, contradiciéndola por momentos, con nostalgia solamente por el "país de la infancia" (132) y -reflexionando sobre los muchachos de su generación, muertos, desaparecidos o que viajaron- el sujeto poético sufre porque no puede integrarse a lo que, con la terminología citada al principio de este párrafo, llamaríamos un que, en realidad, no parece vislumbrarse por ningún lado. Opta entonces por el viaje a los Estados Unidos (si nos atenemos a la imagen de la Estatua de la libertad mencionada en el fragmento VI), pero no lo hace a gusto, sólo mejora con la memoria de la "huaca que nos recompone"(135) -la reivindicación étnica- cuando va saliendo por el aeropuerto.

El segundo compacto Declinaciones latinas empieza así con el sujeto poético ya sentado en el avión "a 33 mil pies de altura" (141). La meditación sobre el problema nacional continúa, pero ahora está trasvasada por una lectura del viaje de Eneas, símil aquí de la fundación de un nuevo lugar para vivir, es decir, en los Estados Unidos, aunque sea "difícil encontrar entre estos almacenes / aromas de lisura y flores de canela" (149), alusión directa al famosísimo valse peruano de Chabuca Granda, en tanto emblema de una tradición peruana que inexorablemente se iría perdiendo. Y solo habría "Corporatio" (149), nada menos que el capitalismo transnacional corporativo de nuestros días, plenos de profunda alienación: "un grupo de señoras habla de las elecciones /como si se tratara del último perfume" (149), lo cual genera un hondo desengaño en la sensibilidad del poeta refugiándose -una vez más- en "la clara conjunción de formas / en la fusión de los más finos elementos" (150) los predios del arte y la creación, entendemos, pero -y he aquí una valiente y sincera confesión de parte- "El poeta viajero se convierte / muy fácilmente en buitre" (152). Sin embargo vendrán "nuevas piernas y sonidos, nuevas consideraciones" (152) aunque finalmente "se concedan doctorados enviadiables / en la ciencia triunfante / de la buitrología..." (153). No habría salida, al parecer, mas existe siempre una salvación por el camino de la poesía: "el poema como el aeroplano / encima de las moscas, pero aún debajo de la Luna” (155). Todavía nos obsede la búsqueda y contemplación de esa belleza. 
Sakra Boccata (2006) es un libro en cuyo centro fulgura el sexo de la mujer abiertamente colocado. Pero no se trata solamente de un erotismo en poesía, sino de una carnalidad fusionada a la experiencia religiosa. El texto inicial reza: "Te soñé todas las noches por más de 300 años” (¿Alusión al entero tiempo de la conquista y época virreinal que nos trajo el catolicismo? No olvidemos que Mazzotti es un experto colonialista), y prosigue: "contaba las sortijas del rosario hasta quedar dormido" (161). Para concluir: "Y las olas martillean Tu Nombre, chorreando por la espuma el néctar / duraznero de tu Sakra Herida, / La alegría de las catacumbas, la resurrección de los muertos / Y la Vida Eterna" (161). Pero la religiosidad en nuestro poeta no es sólo cristiana, sino greco-latina: "Llueve en los montes de Cibelia" (162) arranca el segundo canto aludiendo a la mitológica diosa Cibeles; y es también literaria, textual peruano-poética, diríamos, ya que el verso "Ha llegado la diosa ambarina" (162) -en este mismo poema- junta en un único intertexto la sala ambarina de J.M. Eguren y la divinidad ambarina de E.A. Westphalen. En el canto 3 se define el objeto del deseo: "Tu Koncha es el lugar exquisito más dentro de la guerra" (163) y en esta parte se lo describe con poesía de excelente factura: "Mira el rosado de su pliegue / Como el labio que cubre el horizonte / Al levantar la niebla (163).

En el plano del lenguaje se destaca el trabajo con la aliteración (lo que acerca este libro a la escritura neobarroca latinoamericana). En el texto 4 encontramos -después del trílcico "Vusco Volver" (164)- esta línea: "Vusco tu rosca hosca" (164) "o tu perfil de Lemnia de lunática" (164). Y en el sexto poema preludiado por una cita del gran brasilero Manuel Bandeira: Eu canto assim / como que eu choro de donde Mazzotti toma este verbo que significa lloro y que por capricho idiomático peruano podemos interpretar tambien como choro = ladrón, otorgándole el sentido gongorino de robador al amante que le hurta el amor a la amada y así mismo en el siguiente verso: "Choro porque este chanto es más dulce que la melodía de los cardenales" (166) podemos relacionar este chanto no sólo como canto, sino como el chanto = chantarse del habla peruana, indicando la posición pasiva del acoplamiento sexual y que en términos clásicos correspondería a la entrega femenina, aparte de la la más directa alusión de choro (molusco) al 
sexo femenino. Toda esta intromisión en la jerga nacional no es óbice para que el poeta - ese orfebre que en él habita- nos deje escuchar esta mesurada y preciosista expresión: "se abre a escondidas / Su aroma de sándalo su dulce /[...] Asienta las espaldas sobre el nácar / Permite contemplar la perfección de sus líneas” (166).

Hacia el canto séptimo la hasta ahora innominada musa adopta un apelativo: "LoKilla" que viene del afectuso loquita, pero tambien y por la ortografía incidental que separa la palabra después de la primera sílaba -Killa-que quiere decir luna en el idioma quechua, un llamado hacia el cuerpo satélite, emblema del amor loco de vasta tradición en la literatura universal, pero que aquí gana una raigambre amerindia. Y paradójicamente hacia el final del libro -poema 27reaparece como "Low Killa" (167) con una partícula inglesa que atañe bajo, o lo que está abajo ; sería entonces Baja Luna ya sea en el imperativo del verbo, o haber logrado que la luna descienda hacia uno, o el nombramiento del sexo femenino como esa luna que esta allí abajo. Cabe señalar que Mazzotti toma a la plateada esfera como uno de los leit-motiv centrales del poemario, y desde allí se desencadena la propulsión erótica. En el poema 8 hay una elaborada construcción entre la luna, las olas y su relación con la naturaleza entera, la playa o un árbol "Que tarde o temprano volverá a las aguas / Para posarse en la duna más dorada/ Chorreando su luz" (168). Hablando de la naturaleza y como no podía dejar de ser - canto 9- Mazzotti alude a la flor-símbolo de la poesía identificándola con el sexo femenino: "Rosa pulposa de todos los señoríos" (169), pero inmediatamente la parodia a través de la cita de un conocido valse criollo: "(169) y en los versos finales interviene la letra del difundido bolero "Bésame mucho". Dice el poeta "como si fuera esta noche / La primera vez" (169) y no la última, de aquella canción mexicana. Esto nos permite marcar la diferencia que opera la poesía cuando trabaja con elementos de la cultura popular latinoamericana-desde el acervo musical hasta noticias periodísticas- muchas veces subvirtiéndolos para llegar a nuevas propuestas significativas. De allí que desfilen por el poemario íconos religiosos populares como la Virgen de Chapi o la Virgen de la Candelaria, pero re-significados, mezclados o fusionados por la inspiración del poeta. 
Altamente literario, en este libro campea el intertexto. Desde el ya citado Vallejo, de quien - poema 10- se invierte un verso del super conocido poema Masa. "Tanta muerte y no poder nada contra la vida" (170) reza Mazzotti; hasta el mito órfico del descenso a los infiernos. Pero nuestro poeta -texto 18- le da un toque cotidiano e íntimo: "El Infierno, Eurídice, es tu ausencia / Sobre la faz de la tierra" (175). Es decir, el dolor humano se debe a la ausencia de amor, implicado aquí en la belleza desaparecida de la semidiosa. En este orden de cosas -el intertextual-el poema 20 constituye íntegramente un remake del gran vanguardista bonaerense Oliverio Girondo que por su desopilante ritmo y encabalgamiento sintáctico es para mí una pieza de antología.

En 2009 Mazzotti publicó Las flores del Mall -jugando burlescamente con el fundamental libro de Baudelaire- para reunir un grupo de poemas sobre los Estados Unidos y cifrando su sentido en los insoslayables grandes centros comerciales norteamericanos. La primera parte, "D.C." (District of Columbia), realiza un viaje poético por la capital del país y sus sitios emblemáticos, particularmente el "Independence Mall", esa larga explanada que une el "Lincoln Memorial" con el Capitolio. El poeta comenta -de objetivo modo- la grandeza histórica de los Estados Unidos, su innegabla preeminencia actual en el mundo, pero al mismo tiempo plantea una crítica frontal a diversos aspectos de dicha historia; por ejemplo, las matanzas y masacres de aldeas enteras durante la guerra de Vietnam en los 60s, o el genocidio de las naciones indias; lo cual lo lleva a una posición desesperanzadora. En un poema de "Nueva Albión" -segunda parte del librodirigiéndose a George Washington le expresa "El esplendor de la Gloria, que el pobre hombre imaginara, Jorge, qué decirte / El árbol que pensaste ampararía / La libertad de los humanos, es hoy un adorno / De un cementerio aledaño" (206). Podría resultar claro que el poeta termina por disgustarse abiertamente por la condición tan alienada del sistema y sólo le queda contemplar a las "Muchachas", como se titula el poema final de dicha sección segunda, en donde -en nítida defensa de la escritura, de la opción por la belleza y el erotismo- leemos: "Queda el silencio de la calle anaranjada, queda / El rastro de una oruga desmayada, y 
en los ojos / De la ardiente muchacha por miseria / De líquido semiótico en las venas / Un pálido y oscuro caminante / Que acaso con la lengua la pintaba” (214). Esa sería la function del poeta. Cierra el poemario la parte tercera compuesta por Ernesto E. López - alter ego del autor- denominada "Inmortal (vna dozena de prosas)". Encriptado en el capricho ortográfico y el homenaje a la Muerta Inmortal vallejiana, este conjunto representaría una reflexión sobre el amor y la vida más allá de la muerte. Circulamos por una cárcel o por el infierno -que vienen siendo lo mismo- para ser definidos una vez más y de forma rotunda por la creación literaria: "como un barco iluminado deslizándose bajo el Puente / escribir / De ti, escribir de mí / escribir / De nada, pero / Escribir/ Escribir/ Escribir" (225).

El último libro de nuestro poeta -cerrando esta recopilación El Zorro y la Luna. Poemas reunidos 1986-2016- es Apu-Kalypso / palabras de la bruma (2015). Desde el nombre podemos percatarnos que este conjunto está basado en la fusión. En efecto, Apu es la voz quechua que designa la ancestral divinidad mitológica de las grandes montañas andinas. Mazzotti la junta aquí con Kalypso -la ninfa de la Odisea- y de ambas saldría una aproximación a la cristiana Apocalipsis. Y esto porque -en la visión ecologista que anima el poemario- sería el destino de la humanidad si seguimos el sistemático proceso destructivo de la naturaleza, motivado - por supuesto- por las "manías monetarias" (236) que están a la orden del día en el mundo actual. Toda esta -digamos- "ideología" está sustentada en una composición poética de estilo neobarroco, con lo cual Mazzotti se integra al movimiento quizá mas interesante y renovador de la poesía latinoamericana de hoy. Leamos - por ejemplo- esta conseguida aliteración en "Amazonas", poema que lleva el nombre del gran río sudamericano: "Sabanas sabrosas de savia soberbia de subidas" (237). O en "Glaciares": "resplandor de tus rocas radiantes" (240). Y con étnica sonoridad, en el texto "Sabanas": "La soberana sabana que relumbra su zamba sombra" (255). La fusión está planteada también como entrega erótica de la naturaleza en sí misma -y en ella- la poesía; es decir, el escribir en tanto acto carnal que simultánemante -permítasenos expresarlo de este modo- hace el amor a natura, entidad femenina: "Así desapareces en la bruma 
encendiendo cien ojos / El cielo toca por primera vez la hondura infinita / Goza la gloria tu ligera turbulencia que sólo dice / Las letras de un vacío cuando ya eres un recuerdo" (262). Ante la nada: la memoria del poema.

José Antonio Mazzotti pertenece a la generación de 1980 peruana, aquella cuyos miembros crecimos durante el estado de bienestar que significó la Revolución Peruana del General Velasco, quien encabezó un proceso de reformas estructurales que cambió la historia de nuestro país entre 1968 y 1975. Dicho proceso fue la culminación de un devenir de lucha y anhelo que abrigaba el pueblo peruano desde la propuesta de José Carlos Mariátegui en 1930 y que tuvo un punto de gran efervescencia continental con el triunfo de la Revolución Cubana en 1959. La gesta heroica del Che Guevara -muerto en acción, Bolivia 1967- produjo un efecto de profunda esperanza, así como la difusión masiva de los escritos de Mao Tse-tung en aquellos días. Los poetas peruanos de la generación de 1960 (Cisneros, Hinostroza) pagaron tributo a la Revolución Cubana y cambiaron el terno por él, como dijo alguna vez Antonio Cisneros. Igualmente, su lenguaje conversacional fue extremado por los autores de Hora Zero en 1970 (Pimentel, Ramírez Ruíz,Verástegui), mientras los poetas de nuestra generación buscamos radicalizar aún más dicho lenguaje, ya sea por la vía de la jerga y los espacios de la droga, o una suerte de conceptualismo proclive a la elaboración supermental, como es el caso de Mazzotti. Todo esto alumbrados por los apagones y atentados casi cotidianos de a partir de 1980 .

Ya en los días que corren, el camino ha tomado un rumbo neobarroco, siempre con la opción pura por la poesía, como la única visible e invisible luz al fondo del túnel en que sobrevivimos.

[4 de febrero de 2018, junto al río Cooper, New Jersey South]

\section{Obra citada}

Mazzotti, J. A. (2016). El Zorro y la Luna. Poemas reunidos 1981-2016. Salem, Lima, New York: Editions y Academia Norteamericana de la Lengua Española 